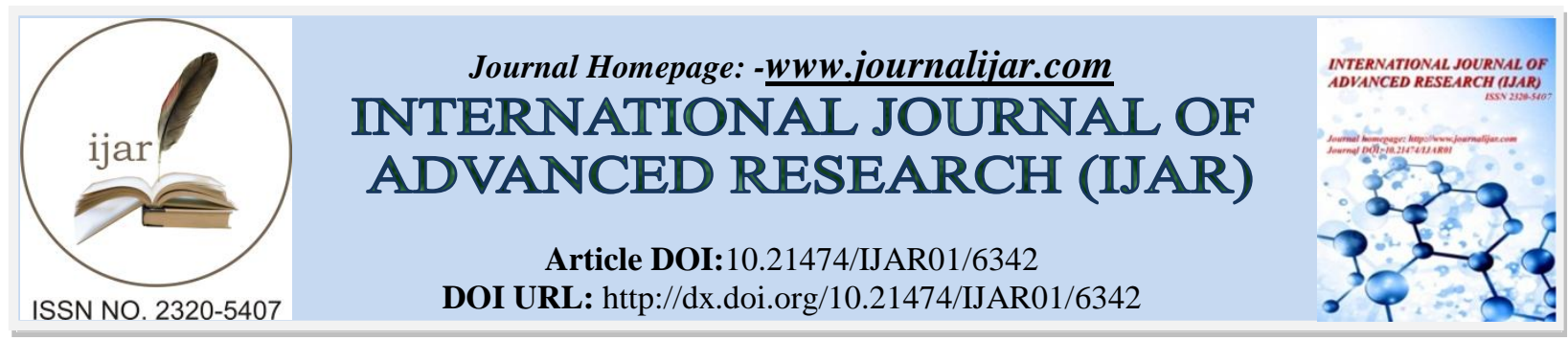

RESEARCH ARTICLE

\title{
THE IMPACT OF LIPOPROTEINS ON HEMORHEOLOGICAL PARAMETERS IN DIABETIC PATIENTS WITH CARDIOVASCULAR DISEASES.
}

Hayder A. L. Mossa ${ }^{1}$, Nabil A. Antwan² and Hashim M. Hashim ${ }^{3}$.

1. High Institute for Infertility Diagnosis and ART's,Al-Nahrain University, Baghdad-IRAQ.

2. Medical College,University of Limerick, Limerick-IRELAND.

3. College of Medicine, Al-Nahrain University, Baghdad-IRAQ.

\section{Manuscript Info}

Manuscript History

Received: 18 November 2017

Final Accepted: 20 December 2017

Published: January 2018

\begin{abstract}
In cardiovascular diseases the plasma lipoprotein changes have an effect on blood flow, so the alterations of the blood and plasma lipoproteins affect the rheological properties. This study included eighty diabetic patients with cardiovascular diseases and seventy healthy controls and were evaluated by measurement the level of lipoproteins \& hemorheological parameters (Blood flow parameters) as plasma viscosity \& RBCs aggregation, all patients and controls were evaluated also by clinical examination and investigation, the study showed that the lipoproteins level in diabetic patients with cardiovascular diseases is highly associated with elevated plasma viscosity \& RBCs aggregation .
\end{abstract}

Copy Right, IJAR, 2018,. All rights reserved.

\section{Introduction:-}

In cardiovascular diseases the plasma lipoprotein changes have an effect on blood flow ,so the alterations of the blood and plasma lipoproteins affect the rheological properties , and play a role in accelerating the rate of progression of complications such as microangiopathy by raising the plasma viscosity and by increasing the affinity of erythrocytes for each other(1), the changes in viscosity and in red -cell aggregation both are the consequence of abnormal plasma lipoproteins, the incidence of which is largely independent of the onset and duration of disease(1).

Subjects and Methods:-

A full history and clinical examination was carried out for all diabetic patients with cardiovascular diseases \& control subjects by physician in AL-Kadhimia Teaching Hospital-Consultant Clinic, the research was carried out in laboratories of clinical physiology department in college of medicine at Al-Nahrain university from November 2002 till December 2003, the subjects were usually fasting for 12 or more hours, $10 \mathrm{ml}$ of blood was aspirated, $4 \mathrm{ml}$ put in an EDTA containing tube(for plasma viscosity \& RBCs aggregation estimations), then centrifuged by centrifuge for 5 minutes at $3000 \mathrm{rpm}, 2 \mathrm{ml}$ of it was stored in the refrigerator for 24 hours to be used for plasma viscosity estimation.

The other $2 \mathrm{ml}$ was put in a small tube and make a mixture consists of $900 \mu 1$ of plasma and $100 \mu \mathrm{l}$ of RBCs and then some of this mixture was taken in a cuvette until the height of $40 \mathrm{~mm}$ in the cuvette, the latter was ready for RBCs aggregation estimation by Laser scattering procedure.

The rest of the blood sample $(6 \mathrm{ml})$ was used for the Lipid profile estimation. 
For the estimation of plasma viscosity a red cell pipette which it considered as simple viscometer was employed for this process(2).

Aside from availability, the red blood cell pipette has several additional advantages including case of filling and cleaning, requirements for only $1 \mathrm{ml}$ of sample and rapid flow time (nearly 90 seconds for water, 2-3 minutes for normal plasma).

It was filled by pulling the sample into the pipette from below, with suction applied through the standard piece of tubing supplied with red cell pipettes.

The time of flow between the lines immediately above and below the bulb reservoir was recorded.

The determinations were carried out at room temperature $\left(23^{\circ} \mathrm{c}\right)$, the instruments were thoroughly rinsed with saline, then distilled water, and finally dried with acetone.

Thorough cleaning is imperative since dirt or dried proteins will impede flow and give falsely high values.

The viscosity of the plasma is calculated from a comparison of its time of flow with that of a fluid of known viscosity (like water which it equals 1), under the same standard conditions (3).

Viscosity of plasma $=($ Viscosity of standard $*$ time of flow of plasma $) /($ Time of flow of standard $)$

For RBCs aggregation estimation the laser scattering method was employed for this study, from scattered light intensity profiles continuously obtained during aggregation of erythrocytes and sedimentation of the aggregates ,characteristic kinetic parameters were computed.

The progression of erythrocyte aggregation and the structure of the aggregates were dependent on both erythrocytes properties and the macromolecules in the medium which is the plasma of the subject(4).

The $10 \%$ PCV suspension that was gently poured into the cuvette is fixed in between the laser beam from Helium -Neon $632.8 \mathrm{~nm}$ which was switched on for 10 minutes prior to the data collection, and the photo cell that recorded the intensity of the transmitted light \& determined automatically with a polygraph. The transmitted light is changed with the progress in aggregation process, the test of recording the aggregate process was repeated again for normal and patient samples.

On the basis of signal pattern and microphotographic observations, three phases were differentiated and the kinetic parameters (times and rates) were defined (Figure 1).

\section{A-Phase 1:-}

In this smooth phase, the signal intensity slightly increased .The time at which the clear fluctuation began to appear was termed as aggregation time (AT).

The rate of aggregation (RA) was obtained from the slope of this phase. This fluctuating signal continued to increase exponentially and reached a maximum transmittance level, and this time was termed as sedimentation time (ST). 


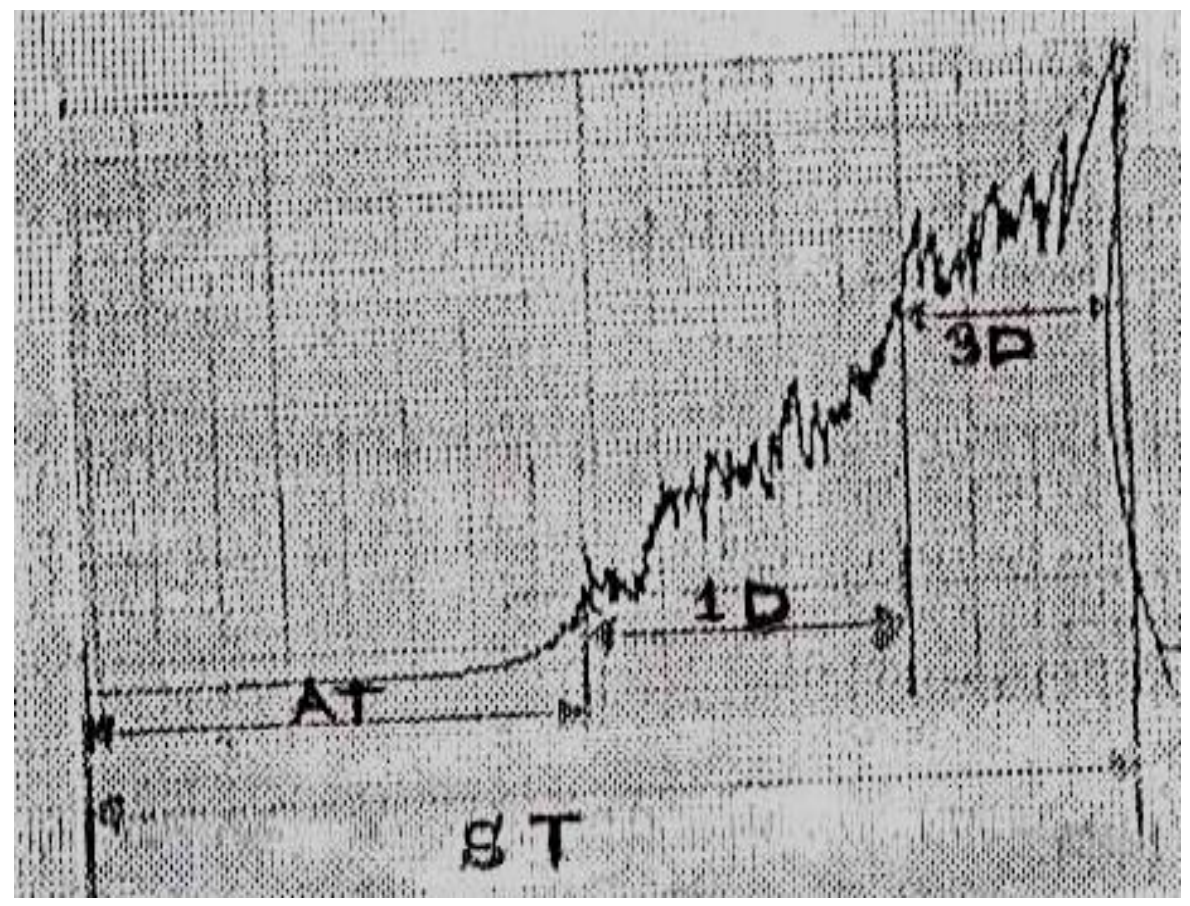

Figure 1:-Three phases are distinguished in a typical pattern of light scattered transmission through the process of aggregation and sedimentation of $10 \%$ erythrocyte concentration in plasma

\section{B-Phase 2:-}

In this phase, the fluctuation of signal intensity was clear and regular, the amplitude was relatively small ,and the mean signal intensity gradually increased .

The interval of second phase was termed as one-dimensional aggregation time (1DAT).

The slope of this phase was termed as rate of sedimentation of one-dimensional aggregates (R1DS).

\section{C-Phase 3:-}

In this phase, the fluctuation was rapid and irregular, and the amplitude was fairly large.

The interval of this phase was termed as three-dimensional aggregation time (3DAT), and the rate of sedimentation of three -dimensional aggregates (R3DS) was obtained from slope of this phase.

The hematocrit of erythrocyte suspension at $10 \%$ is selected to allow normal interaction of various constituents.

So the chamber width is kept sufficiently large to allow uninhibited growth of aggregates and their movement away from chamber walls (5).

These fluctuations could not be observed at the physiological hematocrit $(35 \%-45 \%)$ due to the multiple scattering of light (6).

Light scattering technique has the advantages of quickly providing distinct profiles for different samples with significantly varied kinetic parameters of different phases. Moreover, these profiles and the kinetic parameters can provide a better picture of the interactions of macromolecules like lipoproteins in forming aggregates of erythrocytes and in their sedimentation.

Therefore, these kinetic parameters can be effectively employed to quantify the rheological behavior of erythrocytes in pathological conditions such as cardiovascular diseases \& Diabetes Mellitus. 


\section{Results:-}

It found in this study that the triglycerides were significantly increased compared to the control subjects (Table1). The patients in this study exhibited a high significant increase in the atherogenic index compared to the controls (Table1). The plasma viscosity was increased in diabetic cardiovascular patients \& highly associated with the increase in the lipoprotein level; however this increase was not significantly correlated to the different levels of the lipoproteins (Table1). From the data on RBCs aggregation it is apparent that the diabetic cardiovascular patients showed a shorter aggregation \& sedimentation time\& longer aggregation rate (Figure 2-a, b) which indicates a higher tendency for aggregation. The increase in aggregation rate \& the decrease in aggregation time was highly associated with the increase in the level of lipoprotein (Table1), however this increase \& decrease was not significantly correlated to the different levels of lipoprotein.

Table 1:-Association of the aggregation time, rate \& level of triglyceride \& atherogenic index.

\begin{tabular}{|c|c|c|c|}
\hline Hemorheological Parameters & $\begin{array}{c}\text { DIABETIC PATIENTS WITH } \\
\text { CARDIOVASCULAR DISEASES }\end{array}$ & CONTROL & P-VALUE \\
\hline PLASMA VISCOSITY & $2.202 \pm 0.47$ & $1.746 \pm 0.226$ & $\mathrm{p}<0.0001$ \\
\hline ATHEROGENIC INDEX & $3.524 \pm 1.173$ & $2.401 \pm 0.686$ & $\mathrm{p}<0.0001$ \\
\hline $\begin{array}{c}\text { SERUM TRIGLYCERIDE } \\
\text { [MG/DL] }\end{array}$ & $197.75 \pm 96.56$ & $145.1 \pm 51.15$ & $\mathrm{p}<0.0001$ \\
\hline AT [MIN.] & $6.127 \pm 1.032$ & $6.807 \pm 2.102$ & $\mathrm{p}<0.01$ \\
\hline 1DAT [MIN.] & $2.187 \pm 0.566$ & $2.382 \pm 0.702$ & $\begin{array}{c}\text { Not significant } \\
\mathrm{p}>0.05\end{array}$ \\
\hline 3DAT [MIN.] & $2.029 \pm 0.504$ & $2.231 \pm 0.591$ & $\mathrm{p}<0.028$ \\
\hline RA [MM/MIN.] & $0.362 \pm 0.172$ & $0.324 \pm 0.138$ & $\begin{array}{c}\text { Not significant } \\
\mathrm{p}>0.05\end{array}$ \\
\hline R1DS [MM/MIN.] & $14.11 \pm 3.593$ & $11.89 \pm 3.575$ & $\mathrm{p}<0.0001$ \\
\hline R3DS [MM/MIN.] & $16.51 \pm 4.382$ & $13.47 \pm 2.444$ & $\mathrm{p}<0.0001$ \\
\hline
\end{tabular}
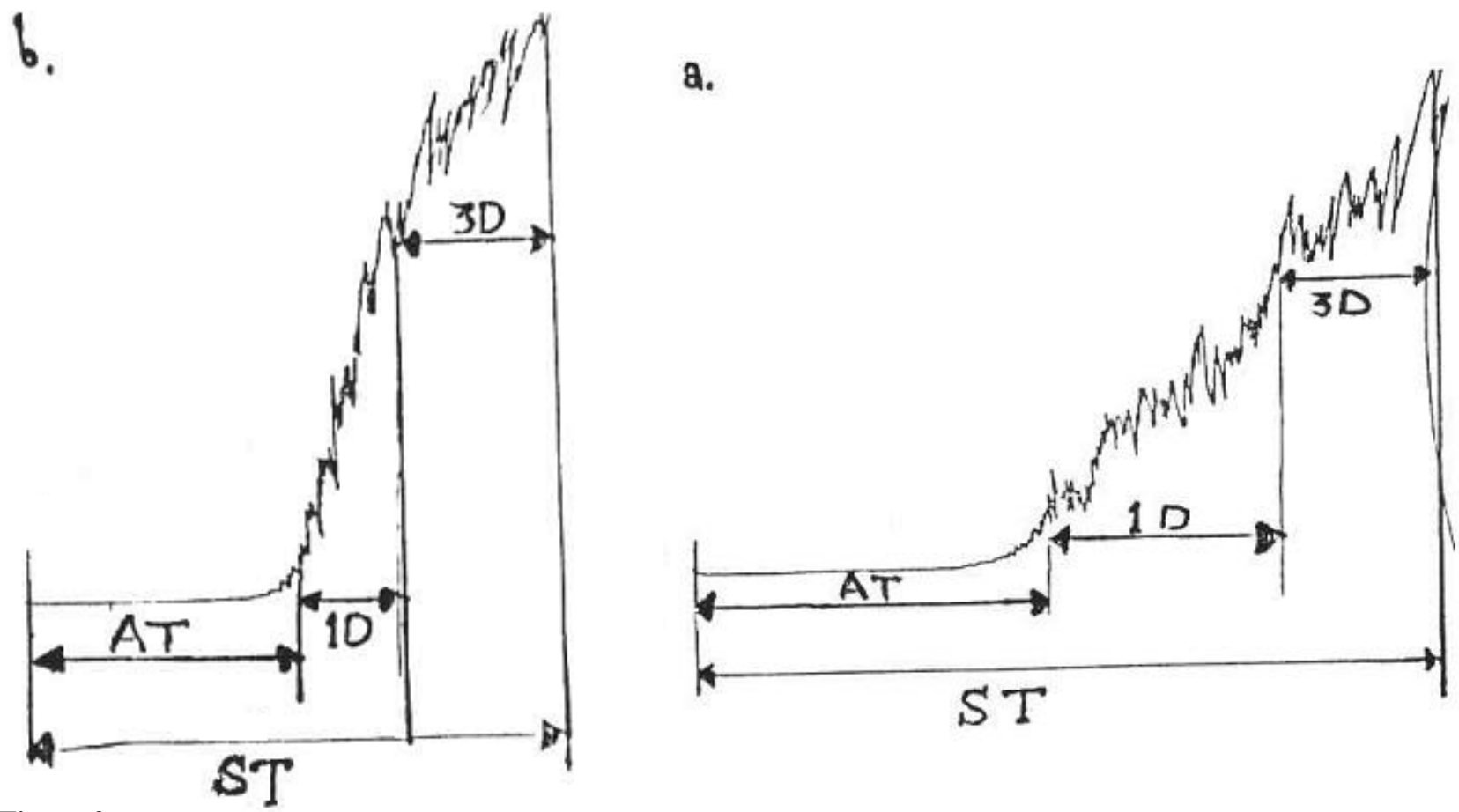

Figure 2:-

a. The pattern of aggregation recorded from a sample of a control subjects.

b. The pattrern of aggregation recorded from a sample of a diabetic patient with cardiovascular diseases. 


\section{Discussion:-}

In diabetic patients with cardiovascular diseases the triglycerides was significantly increased compared to the control subjects.

Same features were found of the uncontrolled glycemic state in other studies $(7,8,9)$ which is characterized by the abnormal lipid metabolism.

The high significant increase in the atherogenic index in diabetic patients with cardiovascular diseases was in accordance with the results of the studies $(10,11)$ where they reported an elevated LDL/HDLC especially in cardiovascular diseases patients with type II Diabetes mellitus.

In this study in diabetic cardiovascular patients the plasma viscosity was increased with a high significant level compared to controls.

This is in accordance with the studies that support the fact that plasma viscosity is elevated in diabetic patients especially uncontrolled patients $(12,1,13,14,15,16,17,18)$. So the metabolic control has a considerable influence on the plasma viscosity. The level of lipoprotein macromolecules was higher in diabetic patients with cardiovascular diseases in comparison to that of the normal subjects, this may suggest that lipoprotein changes are responsible for the increase in aggregation and sedimentation. These are in an accordance with many reports which have emphasized the many factors that may contribute for the increased tendency of aggregation in diabetic cardiovascular patients ,like increased plasma viscosity by high blood glucose, decreased deformability of erythrocytes and increased concentration of lipoprotein macromolecules and their nature $(1,6,19,20,21,22)$.

On the other hand erythrocytes aggregation is a reversible adhesion of adjacent erythrocytes, induced by other bridging macromolecules \& its contribution is uncertain.

\section{Conclusion:-}

The high level of the lipoproteins should be a main factor contributing to the elevation of plasma viscosity in diabetic patients with cardiovascular diseases especially uncontrolled metabolic state ones, and the tendency for RBCs aggregation in diabetic cardiovascular diseases patients together with the elevated plasma viscosity could lead to the disturbance in the circulation through the decrease in the speed of blood flow within the capillaries \& disturb the passage of blood within them that will enhance the formation of sludge that may contribute for the pathogenesis of cardiovascular complications.

So the metabolic control has a considerable influence on pathogenesis \& progression of cardiovascular diseases.

\section{Acknowledgements:-}

We would like to represent our thanks and respects to "Biochemistry Laboratory staff in AL-Kadhimia Teaching hospital" for their great efforts to support, assist, and encourage to accomplish this study.

\section{References:-}

1. Schmid-Schönbein H,Volger E.Red- cell aggregation and Red-cell deformability in Diabetes .Diabetes ,25:897902,(1976).

2. Dorus J. Wright \& David E .Jenkins ,JR. : Simplified method for estimation of serum and plasma viscosity in multiple Myeloma and Related Disorders .Blood Vol.36,No.4(October),(1970).

3. Boroviczeny K G ,Dintenfass L ,Fukada E ,Harkness J ,Lewis S M ,Philips M J,Rewald E ,Ruhenstroth GBauer,Shukla J B , Verwilghen R L ,Seaman GUF, Schmid -Schonbein H, de Clerk F:Recommendation for a selected method for the measurement of plasma viscosity .J clinpathol ,37:1147-1152,(1984).

4. Ethirajan Muralidharan, NorihikoTateishi, Nobuji Maeda. Simultaneous influence of erythrocyte deformability and macromolecules in the medium on erythrocyte aggregation: a kinetic study by a laser scattering technique. Biochimica et BiophysicaActa, 1194, 255-263,(1994).

5. Singh, M. \& Vatsala, T. M. Erythrocyte Sedimentation profiles under gravitational field as determined by HeNe laser.I.Comparison with solid suspensions. Biorheology 19, 165, (1982).[Abstract].

6. Muralidharan, E. and Singh, M. Clin. Hemorheol. 11, 205-216,(1991).

7. Simpson R W, Mann J I, Hockaday J M, Turner R C ,Jelfs R . Lipid abnormalities in untreated maturity onset diabetics and the effect of treatment. Diabetologia, 16: 101-106, (1979).[Abstract]. 
8. Seppo Lehto, Tapani Rönnema, Steven M. Haffner, Kalevi Pyörälä, U cikkokallio, and MakkuLaakso: Dyslipidemia and Hyperglycemia predict coronary heart disease events in middle-Aged patients with NIDDM.Diabetes 46:1354-1359, (1997). [Abstract].

9. Winfried März, Hubert S Charnagl, Claudia Albetshauser, Michael M. Hoffmann, Aloys Berg. Atherogenic Dense LDLs in post menopausal females with the Atherogenic phenotype. Circulation Vol.103, No.15, April 17,(2001).[Abstract].

10. Whitby, L G Smith, A F and Beckett, G J . Disorders of plasma lipids. In: Lecture notes on clinical chemistry .Blackwell scientific publications .Oxford .P.263,(1988).[Abstract].

11. Dasmahapatra ,A,Bale,A.Raghuwanshi,MP,Reddi,A. Byrne ,W. Surarez, S. Nash, H F, Varagiannis , E. and Skurnick, J. Incipient and overt diabetic nephropathy in African Americans with NIDDM .Diabetes care.17 (4).297-304. ,(1994).[Abstract].

12. McMillan D. E., Disturbance of serum viscosity in diabetes mellitus .J.clin.Invest.,53,1071,(1974).

13. Donald E. McMillan, Santa Barbara: Plasma protein changes, Blood viscosity and diabetic microangiopathy .Diabetes 25 (suppl.2):858-64,(1976).

14. Dintenfass ,L.\& Kammer, S.Plasma viscosity in 615 subjects.Effect of fibrinogen ,globulin and cholesterol in normals ,peripheral vascular disease, retinopathy and melanoma.Biorheology,14,247,(1977).

15. Juhan, I. Buonocore, M.,Vovan,L., Durand, F., Calas, M. F., Moulin ,J. P. \& Vague,P. Etude de la filterabilite des hématies chezle diabétique : influence de la glycemie et de 1 hemoglobine A1c. Nouv. Rev. Francais d Hematol. 21, 61, (1979). Cited by E.Muralidharan et al , (1994).

16. Stoltz , J. F. ,Drouin , P. ,Gaillard ,S. ,Rousselle, D.,Vera ,J.C. \&Pointel ,J.P. Variations de la viscosite' sanguino des sujetsdiabétiquesinsulino-dépendants. Ann. Biol. Clin.37,191,(1979).

17. Oughton, J. \& Barnes, A. J. Red cell filterability in diabetes mellitus : its relation other methods of measuring deformability and to blood viscosity. Scand. J. Clin. Lab. Invest. 41,Suppl.156,151-154,(1981).

18. Robert S. Rosenson, Susan Shott, Liping Lu ,Christine C. Tangney . Hypertriglyceridemia and other factors associated with plasma viscosity. The American journal of medicine April 15, 110:488-492, (2001).

19. Ziegler O, Guerci B ,Muller S,CandilorosH,Mejean L ,Donner M et al .Increased erythrocyte aggregation in insulin -Dependent diabetes mellitus and its relationship to plasma factors :a multivariate analysis. Metabolism; 43:1182-1186, (1994).[Abstract].

20. Rogers M,Wiliams R, Rampling W .Decrease in erythrocyte glycophorinsialic acid content is associated with increased erythrocyte aggregation in human diabetes . Clinical science; 82: 309-13,( 1992 ) Cited by Patricia F et al ,(2000).

21. Le-Devehat $\mathrm{C}$,Vimeux $\mathrm{M}$,Bondoux G,Khodabandehlou $\mathrm{T}$. Red blood cell aggregation in diabetes mellitus.Int.Angiol;9:11-15,(1990).[Abstract].

22. Patricia Foresto, Mabel D Arrigo, Larisa Carreras, Raul Etchepare Cuezzo, Juana Valverde, Rodolfo Rasia. Evaluation of Red blood cell aggregation in Diabetes by computerized image analysis .Medicinia (Buenos Aires);60:570-572,(2000). 